\title{
Effect of Flexoelectricity on the Electromechanical Coupling of Piezoelectric Nanoplates
}

\author{
Zheng-Rong ZHANG, Li-Ying JIANG*
}

\author{
Department of Mechanical and Materials Engineering, The University of Western Ontario, London, \\ Ontario, Canada \\ *Iyjiang@eng.uwo.ca \\ ${ }^{*}$ Corresponding author
} Keywords: Flexoelectricity, Piezoelectric Nanoplates, Electromechanical Coupling, Size-dependent
Property.

\begin{abstract}
Flexoelectricity is referred as an instantaneous polarization of dielectrics in response to non-uniform strains or strain gradients. Due to the fact that strain gradients are inversely proportional to the feature size of structures, the flexoelectricity is believed to be significant at the nano-scale. Based on the extended linear piezoelectricity theory, this work attempts to investigate the flexoelectric effect upon the electromechanical coupling of a piezoelectric nanoplate by developing a modified Kirchhoff plate model. Simulation results on the electroelastic fields indicate that the flexoelectric effect is size-dependent, which is more prominent for thinner plates with smaller thickness. It is also observed that the effect of the flexoelectricity upon the electromechanical coupling of the piezoelectric nanoplate is sensitive to the applied electric voltage. This study aims to establish a further understanding of the flexoelectric effect on the electromechanical coupling of piezoelectric nanomaterials, thus provide guidance for the design and applications of piezoelectric nanostructures.
\end{abstract}

\section{Introduction}

The conventional piezoelectricity is a unique feature for noncentrosymmetric crystals, which has been widely explored for applications in transduction technologies. In contrast, flexoelectricity referring to a spontaneous polarization in response to strain gradients, is a universe effect in all dielectrics [1]. Due to the fact that strain gradients typically become manifest for nanostructured materials, the flexoelectricity is believed to be prominent when the characteristic size of the materials scales down to nanometers. Thus the flexoelectricity might play an important role in the size-dependent electromechanical coupling properties of piezoelectric nanomaterials. Owing to the pioneering work in developing theoretical frameworks for dielectrics with the incorporation of the flexoelectricity [2,3], the flexoelectric effect on the physical and mechanical properties of piezoelectric nanostructures could be characterized. Majdoub et al. [4] found that the apparent piezoelectric coefficient of both piezoelectric and nonpiezoelectric nanobeams was significantly enhanced by the flexoelectricity. Chen and Soh [5] demonstrated the effect of the flexoelectricity upon the distribution of the electric polarization in nanocomposite thin films and it was reported that the polarization induced by the flexoelectricity is sensitive to the film thickness. In this work, a modified Kirchhoff plate model will be developed based on the extended linear piezoelectricity theory by incorporating the flexoelectric effect to demonstrate how the flexoelectricity influences the electromechanical coupling behaviors of the piezoelectric nanoplates.

\section{Formulation and Solution}

In the current work, a clamped piezoelectric plate with length $\mathrm{a}$, width $\mathrm{b}$ and thickness $\mathrm{h}$ is considered, which is polarized in the $\mathrm{z}$ direction. According to the Kirchhoff plate theory, the displacements of the plate can be described as 


$$
\varepsilon_{x x}=-z \frac{\partial^{2} w}{\partial x^{2}}, \varepsilon_{y y}=-z \frac{\partial^{2} w}{\partial y^{2}}, \varepsilon_{x y}=-z \frac{\partial^{2} w}{\partial x \partial y} .
$$

Based on the extended linear theory of piezoelectricity with the consideration of strain gradients and polarization gradients, the internal energy density function can be expressed as [3, 4],

$$
U=\frac{1}{2} a_{k l} P_{k} P_{l}+\frac{1}{2} c_{i j k l} \varepsilon_{i j} \varepsilon_{k l}+d_{i j k} \varepsilon_{i j} P_{k}+\frac{1}{2} b_{i j k l} P_{i, j} P_{k, l}+f_{i j k l} u_{i, j k} P_{l}+e_{i j k l} \varepsilon_{i j} P_{k, l},
$$

where $P_{k}, \varepsilon_{i j}$ and $u_{i}$ are components of polarization, strain and displacement tensors, respectively. $a_{k l}, c_{i j k l}$ and $d_{i j k}$ represent the elements of the reciprocal dielectric susceptibility, elastic constant and piezoelectric constant tensors, respectively. $b_{i j k l}, f_{i j k l}$ and $e_{i j k l}$ stand for the polarization gradient and polarization gradient coupling, the strain gradient and polarization coupling and the strain and polarization gradient coupling, respectively.

In general the constitutive equations can be derived from Eq. (2) as [3, 4],

$$
\begin{aligned}
& \sigma_{i j}=\frac{\partial U}{\partial \varepsilon_{i j}}=c_{i j k l} \varepsilon_{k l}+d_{i j k} P_{k}+e_{i j k l} P_{k, l}, \quad \sigma_{i j m}=\frac{\partial U}{\partial u_{i, j m}}=f_{i j m k} P_{k}, \\
& E_{i}=\frac{\partial U}{\partial P_{i}}=a_{i j} P_{j}+d_{j k i} \varepsilon_{j k}+f_{j k l i} u_{j, k l}, E_{i j}=\frac{\partial U}{\partial P_{i, j}}=b_{i j k l} P_{k, l}+e_{k l i j} \varepsilon_{k l} .
\end{aligned}
$$

where $\sigma \mathrm{ij}$ and Ei are the traditional stress and electrical field tensors, while oijk and Eij represent the higher order stress tensor and higher order electrical field tensor, respectively.

For a plate subjected to an electric potential $\Phi$ across its thickness, the electric field in the thickness direction is expressed as [3],

$$
E_{z}=-\frac{\partial \Phi}{\partial z}+E_{z x, x}+E_{z y, y}+E_{z z, z}
$$

In the absence of free electric charges, the Gauss's law requires [6]

$$
-\kappa \Phi_{, z z}+P_{z, z}=0
$$

where $\kappa_{=} \kappa_{0} \kappa_{\mathrm{b}}$ with $\kappa_{0}$ being the permittivity of the vacuum or the air, and $\kappa_{\mathrm{b}}$ being the background permittivity. By using Eqs. (3)-(5) and the electric boundary conditions, i.e., $E_{i j} n_{j}=0, \underset{2}{h} \underset{2}{h}=V$ and $\Phi\left(-\frac{h}{2}\right)=0$ with a voltage $V$ across its thickness, the polarization, the electric field and the stress can be correspondingly obtained in terms of the transverse displacement $w$.

In order to determine the transverse displacement of the plate, Hamilton's principle is adopted to derive the governing equation and the mechanical boundary conditions of the plate [3, 7], i.e.,

$$
\delta \int_{t_{1}}^{t_{2}}\left(-\int_{\Omega} H d \Omega+K+W\right) d t=0
$$

where $H=U-\frac{1}{2} \kappa \Phi_{, z} \Phi_{, z}+\Phi_{, z} P_{z}$ and $K=\frac{1}{2} \iint\left[\rho \frac{h^{3}}{12}\left(\left(\frac{\partial \dot{w}}{\partial x}\right)^{2}+\left(\frac{\partial \dot{w}}{\partial y}\right)^{2}\right)+\rho h \dot{w}^{2}\right] d x d y$ are the electric enthalpy density and the kinetic energy [7] with $\rho$ being the mass density, respectively. When subjected to a uniform transverse distributed load $q$, the work done by both the transverse load and the in-plane forces is $W=\iint q w d x d y-\frac{1}{2} \iint\left[N_{x x}\left(\frac{\partial w}{\partial x}\right)^{2}+N_{y y}\left(\frac{\partial w}{\partial y}\right)^{2}+2 N_{x y}\left(\frac{\partial w}{\partial x} \frac{\partial w}{\partial y}\right)\right] d x d y$ [7].

In the following analysis, we simplify the formulation due to the facts: the strain gradients along the in-plane directions can be ignored in comparison to the strain gradient along the thickness, in which 
the nonzero flexocoupling coefficient is taken as $f_{19}=-e_{19}$; the contracted notation of the material constant tensors is adopted; Accordingly, the governing equation of the nanoplate is derived as [7]

$$
\begin{aligned}
& D_{11}\left(\frac{\partial^{4} w}{\partial x^{4}}+\frac{\partial^{4} w}{\partial y^{4}}\right)+2\left(D_{12}+2 D_{66}\right) \frac{\partial^{4} w}{\partial^{2} x \partial^{2} y}+\frac{d_{31}}{a_{33}} V\left(\frac{\partial^{2} w}{\partial x^{2}}+\frac{\partial^{2} w}{\partial y^{2}}\right)+2 \frac{d_{31} f_{19} h}{a_{33}\left(1+\kappa a_{33}\right)}\left(\frac{\partial^{2} w}{\partial x \partial y}\right)^{2} \\
& -2 \frac{d_{31} f_{19} h}{a_{33}\left(1+\kappa a_{33}\right)} \frac{\partial^{2} w}{\partial x^{2}} \frac{\partial^{2} w}{\partial y^{2}}-\frac{\rho h^{3}}{12}\left(\frac{\partial^{2} \ddot{w}}{\partial x^{2}}+\frac{\partial^{2} \ddot{w}}{\partial y^{2}}\right)+\rho h \ddot{w}-q=0,
\end{aligned}
$$

$$
\begin{aligned}
& \text { with } D_{11} \equiv\left(c_{11}-\frac{d_{31}^{2} \kappa}{\left(1+\kappa a_{33}\right)}\right) \frac{h^{3}}{12}-\frac{f_{19}^{2} h}{a_{33}\left(1+\kappa a_{33}\right)}-\frac{f_{19}^{2} h^{2}}{2 \lambda b_{33}} \frac{e^{\frac{h}{2} \lambda}+e^{-\frac{h}{2} \lambda}}{e^{\frac{h}{2} \lambda}-e^{-\frac{h}{2} \lambda}}+\frac{b_{33} d_{31}^{2} \kappa^{2} h}{\left(1+\kappa a_{33}\right)^{2}}, \\
& D_{12} \equiv\left(c_{12}-\frac{d_{31}^{2} \kappa}{\left(1+\kappa a_{33}\right)}\right) \frac{h^{3}}{12}-\frac{f_{19}^{2} h}{a_{33}\left(1+\kappa a_{33}\right)}-\frac{f_{19}{ }^{2} h^{2}}{2 \lambda b_{33}} \frac{e^{\frac{h}{2} \lambda}+e^{-\frac{h}{2} \lambda}}{e^{\frac{h}{2} \lambda}-e^{-\frac{h}{2} \lambda}}+\frac{b_{33} d_{31}^{2} \kappa^{2} h}{\left(1+\kappa a_{33}\right)^{2}}, \quad D_{66} \equiv \frac{c_{66}}{12} h^{3} .
\end{aligned}
$$

With the aid of Ritz method [8], the governing equation can be solved satisfying the corresponding mechanical boundary conditions for the clamped plate, i.e., $w=0$ and $\frac{\partial w}{\partial n}=0$ at the four edges. Thus the flexoelectric effect on the electroelastic fields of the piezoelectric nanoplate could be characterized.

\section{Results and Discussions}

In the current study, $\mathrm{BaTiO}_{3}$ is taken as the example material with the material constants given in [7]. The plate is subjected to a uniform load $q=0.1 \mathrm{pN} / \mathrm{nm}^{2}$ to ensure the small deformation assumption for the static bending. Fig. 1 plots the variation of the normalized transverse displacement against the plate thickness under different applied electric voltage, in which $w_{0}$ is the displacement without the flexoelectricity. It reveals that the flexoelectricity softens the plate and such an effect is size-dependent. With the plate thickness getting bigger, the flexoelectric effect decays with all the curves approaching unity as expected.

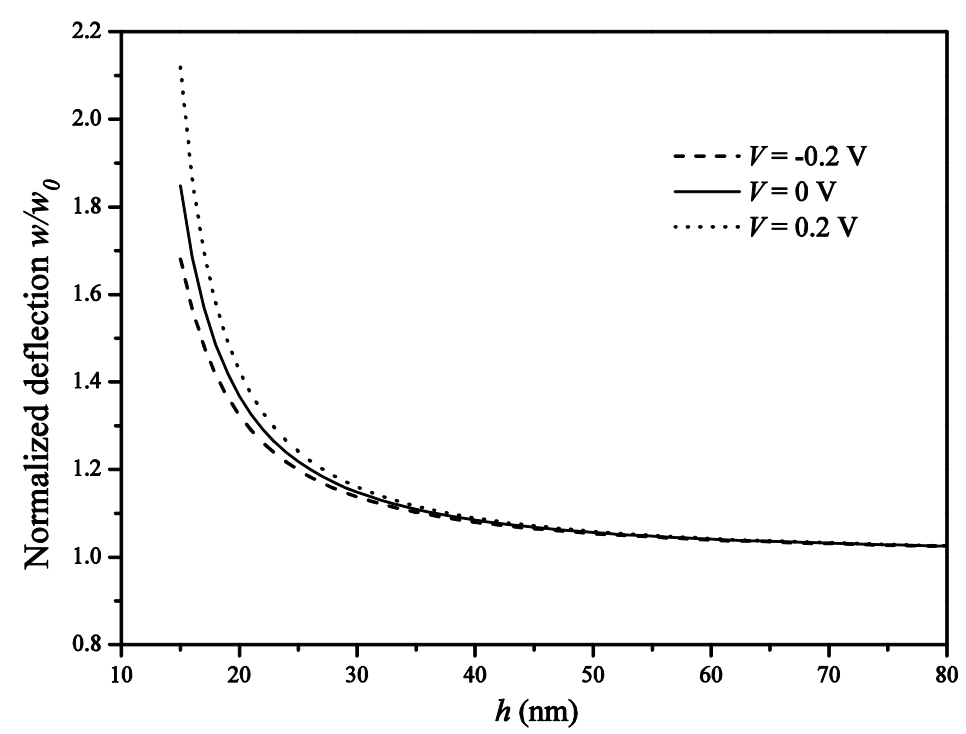

Fig. 1 Normalized maximum deflection versus plate thickness under different voltage $(a=b=40 h)$. 
The variation of the polarization with plate thickness at different location of the plate under various applied voltage is plotted in Fig. 2. As depicted, the flexoelectric effect is more prominent for the plate with smaller thickness. It diminishes with the increase of the plate thickness as the results tend to approach those of the classical Kirchhoff plate model without the consideration of the flexoelectricity. It is also observed that the direction of the applied electric potential influences the flexoelectric effect upon the polarization. For example, for a particular point in the middle of the plate $(x=0.5 a, y=0.5 b)$, the polarization is enhanced by the flexoelectricity with a positive applied voltage. However, the flexoelectricity reduces the polarization at the same point when the applied electric potential becomes negative. It is interesting to point out that such an influence trend is reversed for the other position of the plate, which indicates that the flexoelectricity has an impact on the polarization distribution.
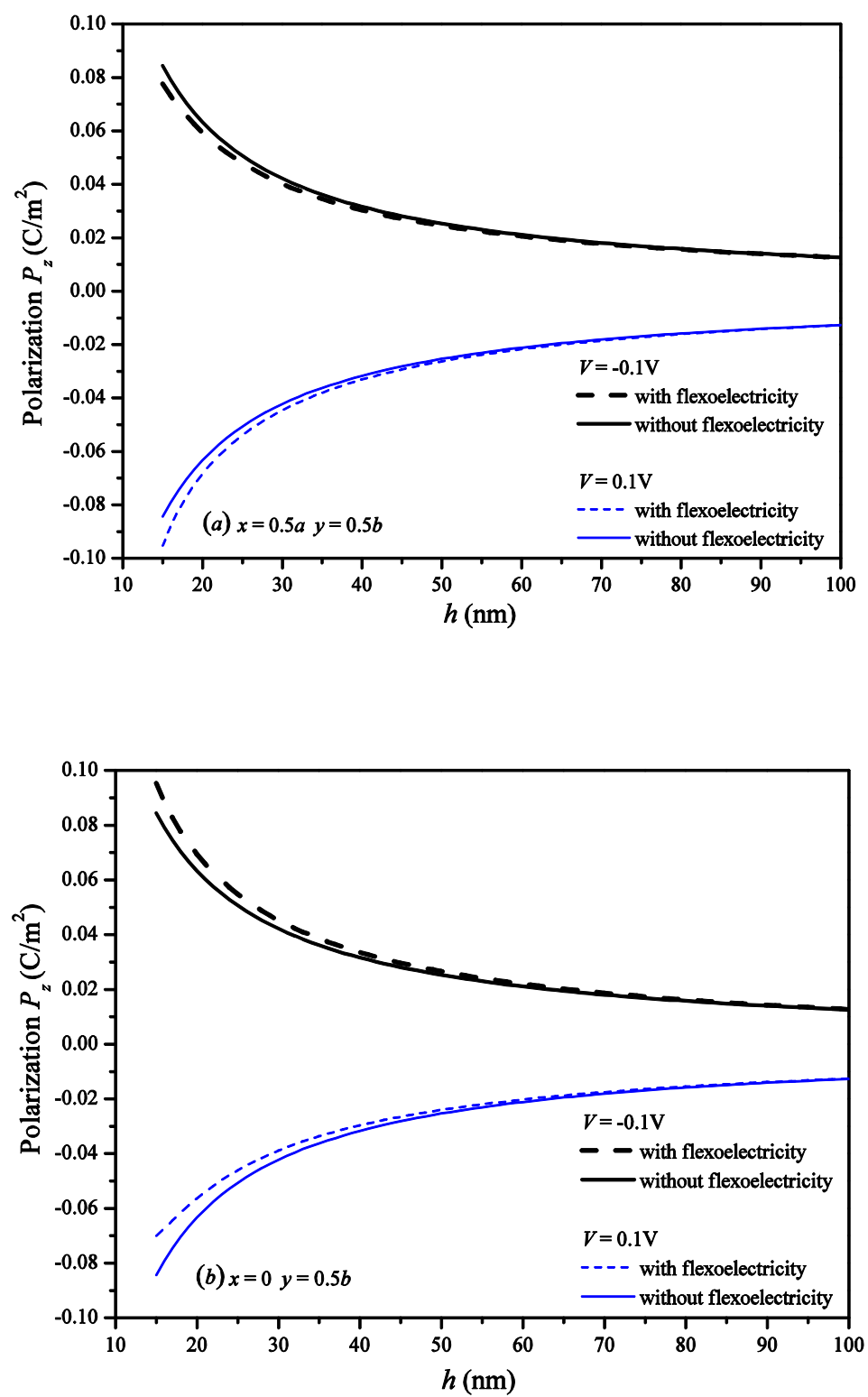

Fig. 2 Polarization versus plate thickness under different voltage at different locations of the plate $(a=b=50 h)$ (a) $x=0.5 a, y=0.5 b$ and (b) $x=0, y=0.5 b$.

The fundamental resonant frequency is plotted in Fig. 3 in order to show how the flexoelectricity influences the free vibration of the piezoelectric nanoplate. $\omega_{0}$ is the resonant frequency of the 
nanoplate without considering the flexoelectricity. As depicted in this figure, the resonant frequency is reduced by the flexoelectric effect which is more pronounced for the plate with smaller thickness, revealing the necessity of considering the flexoelectricity when investigating the free vibration of the piezoelectric nanoplates. It is worth mentioning that the effect of the flexoelectricity is also influenced by the applied voltage as indicated by the discrepancy between the curves, i.e., a positive electric voltage enhances the flexoelectric effect while a negative electric voltage reduces it. The results in this figure implies that the frequency tuning of the piezoelectric nanoplate-based devices through the applied voltage necessitates the consideration of the flexoelectricity.

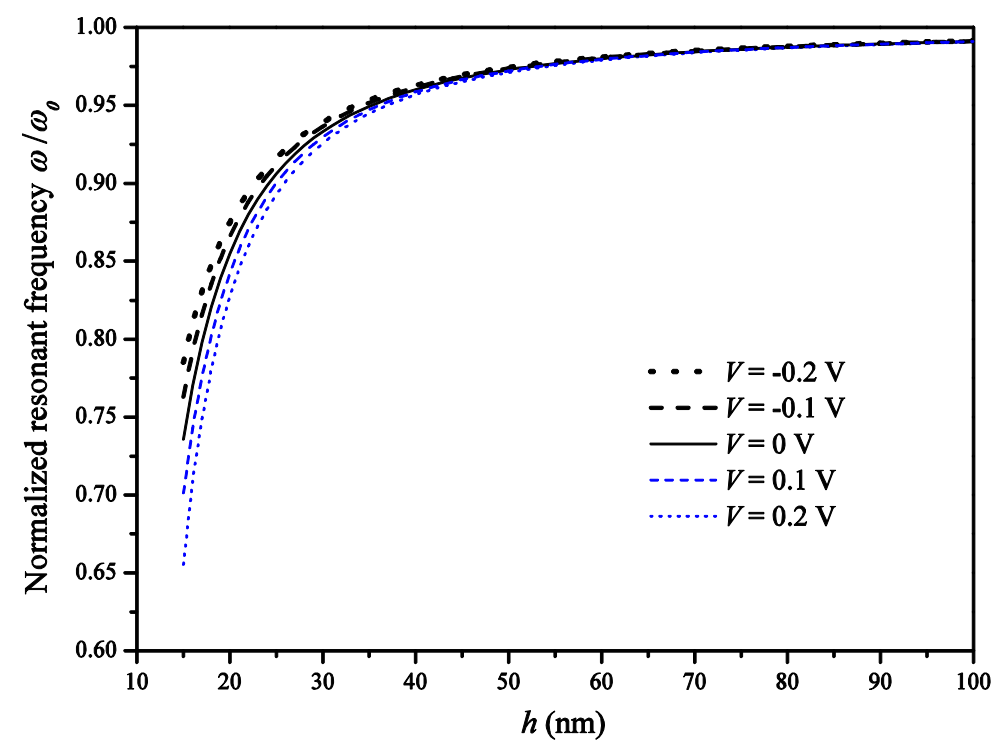

Fig. 3 Normalized resonant frequency versus plate thickness under different voltage $(a=b=50 h)$

\section{Conclusions}

In this work, a modified Kirchhoff plate model based on the extended linear piezoelectricity theory accounting for the flexoelectricity is developed to investigate the size-dependent electromechanical coupling of a piezoelectric nanoplate. Simulation results on both the static bending and free vibration of the plate indicate that such effect is more prominent for thinner plates with smaller thickness and sensitive to the applied electric voltage. The current work is claimed to provide increased understanding on the flexoelectricity and its effect upon the electromechanical coupling of piezoelectric materials.

\section{Acknowledgement}

This work is supported by Natural Sciences and Engineering Research Council of Canada (NSERC).

\section{References}

[1]T.D. Nguyen, S. Mao, Y. Yeh, P.K. Purohit and M.C. McAlpine, Nanoscale flexoelectricity, Adv. Mater. 25 (2013) 946-974.

[2]R. Maranganti, N.D. Sharma and P. Sharma, Electromechanical coupling in nonpiezoelectric materials due to nanoscale nonlocal size effects: Green's function solutions and embedded inclusions, Phys. Rev. B 74 (2006) 014110.

[3] S.L. Hu and S.P. Shen, Variational principles and governing equations in nano-dielectrics with the flexoelectric effect, Sci. China, Ser. G 53 (2010) 1497-1504. 
[4]M.S. Majdoub , P. Sharma and T. Cagin, Enhanced size-dependent piezoelectricity and elasticity in nanostructures due to the flexoelectric effect, Phys. Rev. B 77 (2008) 125424.

[5]H.T. Chen and A.K. Soh, Influence of flexoelectric effects on multiferroic nanocomposites thin bilayer films, J. Appl. Phys. 112 (2012) 074104.

[6]J.Hlinka and P.Marton, Phenomenological model of a $90^{\circ}$ domain wall in BaTiO3-type ferroelectrics, Phys. Rev. B 74 (2006) 104104.

[7]Z.R. Zhang, Z, Yan and L.Y. Jiang, Flexoelectric effect on the electroelastic responses and vibrational behaviors of a piezoelectric nanoplate, J. Appl. Phys. 116 (2014) 014307.

[8] J.N. Reddy, Theory and Analysis of Elastic Plates and Shells, Boca Raton, CRC Press, 2007. 